\title{
ESPECIFICACIONES TÉCNICAS DE BUENAS PRÁCTICAS PARA BOVINOS DE CARNE: UNA BASE PARA EL DESARROLLO SOSTENIBLE Castelán, María Elena ${ }^{1}$
}

\author{
${ }^{1}$ Tesis para optar al grado de Magíster en Gestión Ambiental \\ Facultad de Arquitectura y Urbanismo - UNNE
}

\section{INTRODUCCIÓN}

Las Buenas Prácticas Agrícolas se constituyen en el núcleo de la agricultura moderna al integrar bajo un solo concepto las exigencias agronómicas y las del mercado (FAO, 2003).

En la actualidad más que un atributo, son un componente de competitividad, que permite al productor rural diferenciar su producto de los demás oferentes, con todas las implicancias económicas que ello hoy supone (mayor calidad, acceso a nuevos mercados, consolidación de los actuales, reducción de costos, etc.).

Las BPA pueden simplemente definirse como: "hacer las cosas bien" y "dar garantías de ello". Las BPA son un conjunto de principios, normas y recomendaciones técnicas aplicables a la producción, procesamiento y transporte de alimentos, orientadas a asegurar la protección de la higiene, la salud humana y el medio ambiente, mediante métodos ecológicamente seguros, higiénicamente aceptables y económicamente factibles (FAO, 2004).

La FAO, ha elaborado una definición, más descriptiva y explícita, al señalar que: "consiste en la aplicación del conocimiento disponible a la utilización sostenible de los recursos naturales básicos para la producción, en forma benévola, de productos agrícolas alimentarios y no alimentarios inocuos y saludables, a la vez que se procuran la viabilidad económica y la estabilidad social" (FAO, 2004).

La aplicación de las BPA implica el conocimiento, la comprensión, la planificación y mensura, registro y gestión orientados al logro de objetivos sociales, ambientales y productivos específicos.

Requiere por parte de productores y empresas la adopción de una serie de cambios tecnológicos y metodológicos relacionados con la manera de producir y procesar el producto.

La utilización de herramientas que busquen demostrar mediante procesos adecuados y evidencia de estos, que se están haciendo las cosas correctamente a lo largo de una cadena agroalimentaria. (Peiretti, 2004).
La FAO (2004) menciona como objetivos de las BPA:

- Acrecentar la confianza del consumidor en la calidad e inocuidad del producto.

- Minimizar el impacto ambiental.

- Racionalizar el uso de productos fitosanitarios.

- Racionalizar el uso de recursos naturales (suelo y agua).

- Promover técnicas de Bienestar Animal

- Asumir una actitud responsable frente a la salud y seguridad de los trabajadores.

- La iniciativa de las Buenas Prácticas Agrícolas busca ofrecer un mecanismo para llevar a cabo medidas concretas en pro de la agricultura y el desarrollo rural sostenible.

Los beneficiarios de las BPA alcanzan a los pequeños, medianos y grandes productores agropecuarios, que obtendrían un valor añadido por sus productos y tendrán mejor acceso a los mercados. A los consumidores, que gozarían de alimentos de mejor calidad e inocuos, producidos en forma sostenible. Al comercio y la industria, que lograrían ganancias de ofrecer mejores productos. Y a la población en general, que disfrutaría de un mejor medio ambiente (Guiffré, 2001).

Para lograr esas metas es necesario generar conciencia entre todas las partes interesadas y los gobiernos, en particular los productores y los consumidores, de lo que constituye obtener productos primarios logrados con la implementación de las BPA's en sistemas de producción sostenibles. Los gobiernos y las instituciones privadas deberán formular y aplicar políticas de apoyo. Uno de los incentivos de mayor impacto será obtener precios adecuados por los productos (Viglizzo, 2004).

Por otra parte, la aplicación de las BPA constituye un desafío y una oportunidad para la región, ya que de su cumplimiento dependerá la entrada de sus productos agropecuarios a los mercados con mayor sensibilidad ambiental y creciente exigencia en calidad, ya sean éstos externos o locales. Es este sentido, es necesario que los productores agropecuarios comiencen a interiorizarse seria y rápidamente 
de estos aspectos. Por ello, es menester promover, difundir e implementar programas de BPA para los principales rubros agropecuarios.

El Foro de la cadena agroindustrial argentina (2005) propone entre sus conclusiones difundir, promover e implementar las buenas prácticas y otros sistemas de calidad generando un marco político y técnico adecuado y que involucre a toda la cadena productiva, desde el productor hasta el consumidor. Ese marco deberá ser compartido por el Estado y por el sector privado. Además propone procurar una "visión local" respecto a los sistemas de calidad, sin perder de vista el mercado externo. Esto equivale a generar protocolos propios, adecuados a las condiciones locales e internacionales.

Resulta fundamental entonces la orientación a los productores sin ambigüedades y saber cómo llevar BPA's a la práctica requerirá de un proceso de capacitación de los mismos. Las Guías de Buenas Prácticas tienen un propósito prescriptivo, es decir que ofrece una guía sistemática para orientar la toma de decisiones (Viglizzo, 2004), y ayuda a definir las rutas factibles de decisión, tratando de bajar la incertidumbre tanto como sea técnicamente posible.

Las especificaciones técnicas para alcanzar los objetivos de las BPA deberán estar disponibles para todos los productores como instrumento que facilite la entrada de nuevos productores al sistema. De esta manera obtendrán las bases que se ajusten a protocolos, programas locales o internacionales para la certificación de productos.

\section{MARCO INTERNACIONAL}

El concepto de Buenas Prácticas Agrícolas (BPA) ha evolucionado estos últimos años en el contexto de una rápida modificación y mundialización de la economía de los alimentos y como resultado de los intereses y compromisos de un amplio conjunto de interesados acerca de la producción y seguridad de los alimentos, la inocuidad y calidad de los alimentos y la sostenibilidad ambiental de la agricultura. Entre los principales interesados cabe mencionar a los gobiernos, a las industrias de elaboración y de venta al por menor de alimentos, a los agricultores y a los consumidores, que tratan de alcanzar objetivos concretos de seguridad alimentaria, calidad de los alimentos, eficiencia de la producción, medios de vida y beneficios para el medio ambiente a medio y largo plazo. Las BPA constituyen una forma de contribuir al logro de estos objetivos.

En general, las BPA aplican los conocimientos de que se dispone para lograr la sostenibilidad ambiental, económica y social de la producción y de los procesos posteriores a la producción en la explotación agrícola con el fin de obtener alimentos y productos agrícolas no alimenticios inocuos y sanos. Muchos agricultores de los países desarrolla- dos y en desarrollo ponen en práctica las BPA mediante métodos agrícolas sostenibles como la lucha integrada contra las plagas, la gestión integrada de los nutrientes y la agricultura de conservación. Estos métodos se aplican en un conjunto de sistemas agrícolas y en unidades de producción de diferente tamaño, incluso como aportación a la seguridad alimentaria, fomentados por políticas y programas estatales de apoyo.

\section{APLICACIONES ACTUALES DE LAS BPA}

Los gobiernos, las ONG y las organizaciones de la sociedad civil y el sector privado, están poniendo en práctica las aplicaciones de las BPA para atender a las necesidades de los agricultores y a las exigencias específicas de la cadena alimentaria, pero no de una manera integral y coordinada. En muchos casos la elaboración de BPA en los planos internacional y nacional se ve completada con adaptaciones más concretas para uso en el plano local. A continuación se dan unos pocos ejemplos de aplicaciones actuales.

Los gobiernos, los organismos internacionales y las ONG promueven métodos de agricultura sostenible como la lucha integrada contra las plagas, la gestión integrada de los nutrientes y la agricultura de conservación, entre otras, destinados a mitigar riesgos ambientales y sociales concretos en un conjunto de sistemas de producción y explotación agrícola. La lucha integrada contra las plagas se describe como una práctica recomendada en el Código de Conducta sobre los Plaguicidas (FAO, 2002) y en el Capítulo 14 del Programa 21. Estos métodos que son especialmente adecuados para los agricultores de países en desarrollo, contribuyen a la producción de alimentos, a la seguridad alimentaria local y conservan los recursos naturales.

Los organismos nacionales han promovido las buenas prácticas agrícolas tanto para garantizar la calidad como la gestión ambiental. Entre ellos cabe mencionar los organismos estatales del $\mathrm{Ca}$ nadá, los Estados Unidos, Francia, Letonia, Lituania, Nueva Zelandia, Polonia, el Reino Unido y el Uruguay que han adoptado prácticas adecuadas con respecto al programa agrícola en marcha del Báltico. En Brasil, el EMBRAPA, en colaboración con la FAO, está elaborando una serie de directrices técnicas concretas con respecto a melones, mangos, frutas y hortalizas, frutos del campo, productos lácteos, carne de vaca, carne de cerdo y aves de corral, sobre la base de BPA puestas a prueba por productores en pequeña, mediana y gran escala.

El sector privado, en particular los elaboradores industriales y los vendedores al por menor, utilizan las BPA con miras a lograr una garantía de calidad, la satisfacción del consumidor y beneficios en la producción de alimentos inocuos y de alta calidad 
a lo largo de la cadena alimentaria. Estos esfuerzos incorporan cada vez más criterios de sostenibilidad en respuesta a la demanda del consumidor. Entre otros ejemplos cabe mencionar los Códigos de Prácticas Genéricos EUREPGAP relativos a los productos frescos, la integración de la agricultura y la ganadería; la Iniciativa Agrícola Sostenible (Unilever, Nestlé, Danone y otros); y el Codex Común de Agricultura Integrada de EISA. Unilever ha elaborado "indicadores de agricultura sostenible" más específicos con respecto a los logros de cultivos y emplazamientos específicos (Romano, 2005). La promoción de las buenas prácticas agrícolas por parte de los elaboradores de alimentos y vendedores al por menor puede facilitar la adopción de prácticas agrícolas sostenibles mediante el establecimiento de incentivos por medio de oportunidades potenciales de incorporación de un valor añadido para los agricultores.

Si bien el Codex Alimentarius define de manera concreta las BPA en el contexto del uso de plaguicidas, el Código de Prácticas (Principios Generales de Higiene Alimenticia) y otros códigos más específicos abordan las buenas prácticas en la producción primaria, así como los sistemas posteriores a la producción (Foro De La Cadena Agroindustrial Argentina, 2005).

Como cabe observar en estos ejemplos, las BPA representan una diversidad de enfoques y aplicaciones que tienen en cuenta un conjunto de necesidades en muchas partes del mundo.

La primera dificultad estriba en garantizar que la ampliación del uso de las buenas prácticas agrícolas tenga en cuenta los intereses de los productores en pequeña escala de los países en desarrollo tanto en lo que respecta a la inocuidad como a la sostenibilidad de la producción doméstica.

\section{EN LA ARGENTINA}

El plan ganadero Nacional fue lanzado el 17 de julio de 2006. El plan presentado apunta a una mejora en la producción y en la comercialización sin abordar temas ambientales, la inocuidad y calidad de los alimentos y la sostenibilidad ambiental. Es de suponer que deja estos temas a las provincias y a los técnicos encargados de desarrollar los planes en las distintas zonas del país. Además las realidades en cada provincia son diferentes en lo que respecta a distribución de la tierra, niveles productivos, tamaño de las unidades ganaderas rentables, infraestructura de producción y comercialización, nivel de capacitación de productores. Esto hace que los objetivos y las metodologías para llegar a los productores sea diferente para cada región de la Argentina. En las zonas con mayor fragilidad ecológica pasan a tener mayor jerarquía los objetivos sociales y de sustentabilidad ambiental, apuntando a mejo- rar la calidad de vida de los pobladores, pasando a otro nivel la búsqueda de competitividad a escala internacional.

Casi simultáneamente se inició a través de la página web de SAGPyA una encuesta para iniciar un sistema estandarizado de Buenas Prácticas de Producción Ganadera. En ella solicita colaboración en el desarrollo de ese sistema.

Es la primera iniciativa tendiente a uniformar y estandarizar la producción ganadera. La misma no fue difundida de otra manera lo que indicaría que no forma parte del Plan Ganadero Nacional. Es notable la falta de articulación y estrategias de mejoramiento de la producción primaria en el gobierno. Las provincias del Chaco y Formosa poseen protocolos de carne producida a pasto en donde definen atributos de calidad del producto. Además, ambas provincias pusieron en marcha planes ganaderos que en general tienen los mismos criterios del Plan Ganadero Nacional. Por ello se presenta el siguiente proyecto de gestión de las BPG, que apunta a la articulación entre las provincias que componen el Nordeste Argentino, así como la integración de los eslabones de la cadena de producción bovina, para beneficio de la economía regional conjuntamente con una mejora en la calidad de vida de la población.

\section{OBJETIVOS}

\section{General}

Desarrollar las especificaciones técnicas para alcanzar buenas prácticas ganaderas para bovinos de carne como base del desarrollo sostenible de los sistemas pecuarios

\section{Específicos}

Ofrecer una guía sistemática de operaciones para la toma de decisiones a nivel establecimiento ganadero.

Difundir, promover e implementar las acciones para las BPG para bovinos de carne.

\section{DESARROLLO}

Las BPA constituyen una herramienta cuyo uso persigue la sustentabilidad ambiental, económica y social de las explotaciones agropecuarias, lo cual debe traducirse en la obtención de productos alimenticios y no alimenticios más inocuos y saludables para el autoconsumo y el consumidor.

Se analizan los requerimientos especificados en los convenios y las normas nacionales e internacionales, de adhesión voluntaria así como las obligatorias, para los elementos que intervienen en el proceso de producción ganadera.

\section{Campo de Aplicación}

Los pequeños, medianos y grandes productores, podrán agregar valor a sus productos y tendrán mejor acceso a los mercados. Los consumidores gozarán de alimentos de mejor calidad e inocuos, 
producidos en forma sostenible. El comercio y la industria, obtendrán ganancias al ofrecer mejores productos. La población en general, disfrutará de un mejor medio ambiente.

\section{Términos y Definiciones}

Los términos son mencionados a modo de glosario estableciendo definiciones de acuerdo al uso y legislación de Argentina.

\section{Instalaciones}

Las instalaciones referidas corresponden a: corrales, mangas, balanzas, lugares de confinamiento temporario, cargaderos, potreros, aguadas, alambrados, galpones.

Se detallan las especificaciones referentes a: lugar, ambiente, bioseguridad, condiciones estructurales, superficies mínimas, manejo intensivo, encierre temporal, medidas higiénicas.

\section{Manejo Sanitario y Control de Plagas}

Incluye características de plan sanitario, productos autorizados, almacenamiento seguro, instrucción del personal, vías de aplicación, instrumental adecuado, periodo de resguardo, limpieza de equipos e instrumental, manejo y disposición de residuos y envases, así como el tipo de documentación y registros que debe llevar el establecimiento.

\section{Alimentación y Agua}

La alimentación debe garantizar las características organolépticas y nutritivas de la carne de animales alimentados fundamentalmente con pasto. Mantener el producto final (carne) libre de residuos tóxicos para la alimentación humana. Impedir la posible transmisión de enfermedades al hombre evitando el uso de suplementos prohibidos en la alimentación animal.

Se permite la suplementación estratégica. Si los suplementos son elaborados por terceros se debe establecer las especificaciones de su composición. Se prohíbe el uso de suplementos proteicos de origen mamífero. No se podrán utilizar promotores de crecimiento.

En cuanto al agua debe ser apta para uso animal, libre de residuos y contaminantes. De acuerdo a un análisis de riesgo, se determinará la frecuencia de muestreo y las determinaciones de laboratorio.

Se deberá llevar registro de pastoreo, suplementación, resultados de los análisis de agua.

\section{Transporte de Ganado}

El transporte debe brindar las condiciones necesarias que permitan minimizar el estrés de los animales producido durante la carga, transporte y descarga, para evitar el deterioro de algunas características organolépticas de la carne. Las condiciones higiénicas y de bienestar animal se deben mantener durante esta etapa del proceso productivo.

Se especifican los puntos: condiciones, responsabilidades, duración del transporte, carga y descarga de animales, tipo de documentación y registros necesarios.

\section{Registros e Identificación Animal \\ Identificación del ganado bovino argentino. Tra- zabilidad}

Este sistema, junto con el implementado por el frigorífico, permite identificar el lote o tropa de animales que dio origen a un determinado corte de carne. El "Sistema de Identificación de Ganado Bovino para Exportación" que crea la Resolución 15/2003 del SENASA se aplica en forma obligatoria en todos los campos inscriptos en el "Registro de Establecimientos Rurales Proveedores de Ganado para Faena de Exportación a Unión Europea". Se basa en la identificación de los animales por medio de una caravana, que se coloca en la oreja izquierda, y que debe contener al frente un código no repetible, y al dorso el número del Registro Nacional Sanitario de Productores Agropecuarios (RENSPA) del propietario al cual pertenecen. Estos campos sólo pueden proveer animales de su producción o de campos de cría que, a su vez, estén inscriptos en el "Registro de Establecimientos de Origen" que establece la Resolución 391/2003 del SENASA. De esta forma, se asegura que los inscriptos en esta última normativa, abastezcan de animales a los campos registrados en la Resolución 15/03, garantizando la no utilización de anabólicos o promotores de crecimiento, prohibidos para animales faenados con destino a los países comunitarios.

Se establecen las especificaciones y tipo de documentación y registros que se requiere de cada establecimiento.

\section{Bienestar Animal}

Es el trato humanitario brindado a los animales definiendo a este como "el conjunto de medidas para disminuir la tensión, sufrimiento, traumatismos y dolor de los animales durante su traslado, exhibición, cuarentena, comercialización, aprovechamiento, entrenamiento y sacrificio". Deficiencias en el bienestar animal pueden traducirse por cambios de comportamiento, fisiología, estado sanitario y reproducción o crecimiento.

La American Veterinary Association en 1990 amplía este concepto para incluir que "todos los aspectos de bienestar animal, incluyendo alojamiento apropiado, manejo, alimentación, tratamiento y prevención de enfermedades, cuidado responsable, manipulación humanitaria, y cuando sea necesaria, la eutanasia humanitaria" (De la Sota, 2004). Se detallan las especificaciones de cada uno de estos puntos.

\section{Condiciones de Trabajo de los Trabajadores}

Las especificaciones tienen como objetivos: Dirigir todas las prácticas al logro de un equilibrio óptimo entre aspectos económicos ambientales y sociales.

Proporcionar ingresos adecuados y seguridad alimentaria a las familias.

Adoptar procedimientos seguros de trabajo, horarios aceptables y periodos de descanso. Instruir a 
los trabajadores sobre la utilización segura y eficaz de instrumentos y maquinarias (bioseguridad).

\section{Manejo Medioambiental}

Este aspecto apunta a la correcta recolección, disposición y aplicación de los residuos provenientes de los planteles bovinos. Debe evitar la contaminación de las aguas por escurrimiento y/o por infiltración en el suelo, o arrastre hacia aguas superficiales. Tiene que considerar los efectos en el medioambiente de otro tipo de contaminantes, preservando la biodiversidad.

Se detallan las especificaciones sobre: manejo y empleo de purines y fertilizantes, encierre temporal, manejo de animales muertos, manejo y disposición de residuos y envases, biodiversidad e impacto territorial.

\section{Proyecto de gestión de las Buenas Prácticas}

\section{Ganaderas a nivel regional (NEA)}

Objetivos

Generales:

- La estrategia básica del Programa busca consolidar en primer término una estructura de asistencia técnica que incremente la oferta tecnológica institucional existente, en el marco de una amplia integración entre el sector público y el sec- tor privado, a nivel de los eslabones de la cadena de la producción de carne bovina.

- Acrecentar y sostener la eficiencia de la producción bovina, a partir de los recursos disponibles, para mejorar la rentabilidad y sustentabilidad de las pequeñas y medianas empresas, mediante la generalización del uso la BPG que garanticen calidad e inocuidad alimentaria.

Específicos:

- Motivar al sector productivo primario para generar cambios de actitudes en el gerenciamiento de los recursos y modos de gestión de la producción ganadera.

- Promover a nivel interinstitucional la realización de acciones destinadas implementar las Buenas Prácticas Ganaderas.

- Capacitar a profesionales, dirigentes, productores y trabajadores rurales en orden a acciones y procesos que contribuyan a la aplicación de las BPG.

- Asistir técnicamente, a través de profesionales capacitados, a los productores ganaderos y trabajadores rurales para la implementación de tecnologías que mejoren sus resultados productivos y financieros, y le permitan incrementar en forma creciente la calidad del producto final.

Etapas, acciones y productos del Proyecto

\begin{tabular}{|c|c|c|}
\hline Etapas & Acciones & Producto \\
\hline $\begin{array}{l}\text { Estructura } \\
\text { organizativa }\end{array}$ & $\begin{array}{l}\text { Convocatoria a los actores } \\
\text { involucrados: Gobierno, } \\
\text { Organizaciones de } \\
\text { productores, ONG, etc. }\end{array}$ & $\begin{array}{l}\text { Definición marco de trabajo y } \\
\text { responsabilidades } \\
\text { Consejos de gestión por municipios } \\
\text { Elaboración Programa de difusión } \\
\text { Elaboración de procedimientos e } \\
\text { instructivos }\end{array}$ \\
\hline Programa de difusión & $\begin{array}{l}\text { Capacitación de los agentes de } \\
\text { difusión }\end{array}$ & $\begin{array}{l}\text { Cursos, Talleres, Charlas. } \\
\text { Material escrito, audiovisual, etc. }\end{array}$ \\
\hline $\begin{array}{l}\text { Formación de diferentes } \\
\text { niveles de asociativismo }\end{array}$ & $\begin{array}{l}\text { Asistencia técnica y } \\
\text { capacitación de productores } \\
\text { Aplicación de procedimientos } \\
\text { Control y verificación }\end{array}$ & $\begin{array}{l}\text { Aplicación de las BPG en } \\
\text { establecimientos de productores } \\
\text { Certificación de calidad }\end{array}$ \\
\hline $\begin{array}{l}\text { Gestión de canales de } \\
\text { comercialización }\end{array}$ & $\begin{array}{l}\text { Incorporación de toda la } \\
\text { cadena de producción en la } \\
\text { implementación de las Buenas } \\
\text { Prácticas de Manufactura } \\
\text { Difundir y capacitar al } \\
\text { consumidor sobre calidad de } \\
\text { productos }\end{array}$ & $\begin{array}{l}\text { Implementación de BPM en mataderos } \\
\text { y frigoríficos } \\
\text { Programa de Promoción del producto } \\
\text { Expansión hacia el mercado interno } \\
\text { Expansión al mercado externo }\end{array}$ \\
\hline $\begin{array}{l}\text { Gestión de } \\
\text { financiamiento para la } \\
\text { implementación de las } \\
\text { BP en toda la cadena } \\
\text { productiva }\end{array}$ & $\begin{array}{l}\text { Apoyo financiero del estado } \\
\text { para el desarrollo del plan } \\
\text { Canales de financiación } \\
\text { para pequeños y medianos } \\
\text { productores }\end{array}$ & Planes de financiamiento \\
\hline
\end{tabular}


Plan de actuación según estructura organizativa

\begin{tabular}{|c|c|c|}
\hline & Integrantes & Funciones \\
\hline Grupos de productores & Productores, Técnicos & $\begin{array}{l}\text { Aplicación BPG, comunicación y difusión a } \\
\text { otros productores }\end{array}$ \\
\hline Grupo técnico & $\begin{array}{l}\text { Profesionales de Ciencias } \\
\text { Agrarias, INTA, } \\
\text { Universidades, }\end{array}$ & $\begin{array}{l}\text { Asistencia técnica, Capacitación, Difusión, } \\
\text { Certificación de calidad }\end{array}$ \\
\hline Comisiones Locales & $\begin{array}{l}\text { Gobierno, SENASA, } \\
\text { Organizaciones de } \\
\text { productores, Sociedades } \\
\text { Rurales, ONG, } \\
\text { Comerciantes, Industria } \\
\text { frigorífica, Representante } \\
\text { del grupo técnico }\end{array}$ & $\begin{array}{l}\text { Elaboración Programa de difusión } \\
\text { Elaboración de procedimientos e } \\
\text { instructivos } \\
\text { Control Plan de gestión BPG y BPM } \\
\text { Gestión de canales de comercialización }\end{array}$ \\
\hline $\begin{array}{l}\text { Comisiones } \\
\text { Provinciales }\end{array}$ & $\begin{array}{l}\text { Representantes comisiones } \\
\text { locales }\end{array}$ & $\begin{array}{l}\text { Control Plan de gestión BPG y BPM } \\
\text { Difundir y capacitar al consumidor sobre } \\
\text { calidad de productos } \\
\text { Gestión de canales de comercialización }\end{array}$ \\
\hline Comisiones regionales & $\begin{array}{l}\text { Representantes } \\
\text { Comisiones Provinciales }\end{array}$ & $\begin{array}{l}\text { Gestión de financiamiento para la } \\
\text { implementación de las BP en toda la cadena } \\
\text { productiva } \\
\text { Plan de Desarrollo estratégico regional } \\
\text { Programa de Promoción del producto } \\
\text { Gestión de nuevos mercados }\end{array}$ \\
\hline
\end{tabular}

- Promover, mediante una estrecha cooperación interinstitucional, la creación de las condiciones organizativas, de infraestructura y de servicios de apoyo a la producción y transformación ganadera, de manera de viabilizar mejores condiciones para un volumen creciente del comercio interno y externo de carnes.

- Promover la integración empresarial, entre los diferentes eslabones de la cadena agroalimentaria de la carne, a los fines de concretar operaciones comerciales en conjunto, como también desarrollar estrategias comerciales con actores externos al sector.

\section{Fines}

- Mejorar la calidad de vida de la población.

- Alcanzar la gestión sostenible del medio ambiente.

- Minimizar los riesgos para la salud por la ingesta de alimentos.

- Mejorar la salud y seguridad de los trabajadores.

- Integrar la mayor cantidad de productores a la producción bajo las normas de BPG.

- Lograr prestigio en mercados locales y presencia en los internacionales.

- Alcanzar de mayores ingresos mediante la valorización del producto.

\section{Componentes}

La operación del Programa implica la estructuración de las actividades necesarias para el cumpli- miento de los objetivos propuestos, en siete componentes:
1. PROMOCIÓN Y MOTIVACIÓN
2. CAPACITACION
3. ASISTENCIA TÉCNICA
4. ASOCIATIVISMO
5. CALIDAD
6. COMERCIALIZACIÓN
7. ASISTENCIA FINANCIERA

\section{BIBLIOGRAFÍA}

De la Sota, M. D. 2004. Manual de Procedimientos en Bienestar Animal. Dirección Nacional de Sanidad Animal. SENASA. 38 pp.

FAO. 2003. Elaboración de un marco para las buenas prácticas agrícolas. Comité de Agricultura. $17^{\circ}$ período de sesiones, Roma.

FAO. 2004 Las buenas prácticas agrícolas. Oficina Regional de la FAO para América Latina y el Caribe. 120 pp.

FORO DE LA CADENA AGROINDUSTRIAL ARGENTINA. 2005. Comisión de Las Buenas Prácticas Agrícolas. Diagnóstico y Propuestas. Primer eslabón. Documento de trabajo. 28 pp.

GUIFFRE, L. 2001. Impacto Ambiental en Agroecosistemas. Ed. Facultad de Agronomía. Buenos Aires: 267 pp.

PEIRETTI, R. 2004. El modelo agrícola de CAAPAS. Actas del XII Congreso de AAPRESID, Rosario. 
ROMANO, G. S. 2005. Buenas Prácticas Agrícolas (BPA). INTA EEA INTA Chubut. Boletín $\mathrm{N}^{\circ} 11$. VIGLIZZO, E. 2004. Desarrollo de una metodología compatible con la norma ISO 14000 para la eco-certificación de predios rurales. INTA. Programa Nacional de Gestión ambiental.

\section{Páginas web consultadas}

www.codexalimentarius.net/web/index_es.jsp

www.Eurep.org. EUREPGAP. Buenas prácticas agrícolas y ganaderas. Protocolo aseguramiento integrado de fincas.

www.fao.org/docrep.htm. FAO. 2002. Código Internacional de Conducta para la Distribución y Utilización de Plaguicidas (Versión Revisada) Adoptado por el $123^{\circ}$ periodo de sesiones del Consejo de la FAO.
www.fao.org/docrep/MEETING/006/Y8704S. HTM.

http://www.fda.com FDA -CFSAN. Guía para Reducir al Mínimo el Riesgo Microbiano en los Alimentos en el Caso de Frutas y Vegetales Frescos. 1998.

www.senasa.org.ar SENASA. Legislación.

www.un.org/esa/sustdev/documents/agenda21/spanish/agenda21sptoc.htm Programa 21: Capitulo 14: Fomento De La Agricultura Y Del Desarrollo Rural Sostenibles. 